\title{
Low-noise rotating sample holder for ultrafast transient spectroscopy at cryogenic temperatures
}

\author{
R. Fanciulli and I. Cerjak \\ FOM Institute for Atomic and Molecular Physics (AMOLF), Kruislaan 407, 1098 SJ Amsterdam, \\ The Netherlands \\ J. L. Herek \\ FOM Institute for Atomic and Molecular Physics (AMOLF), Kruislaan 407, 1098 SJ Amsterdam, \\ The Netherlands and Optical Science Group, MESA + Institute for Nanotechnology, University of Twente, \\ Enschede, The Netherlands
}

(Received 19 December 2006; accepted 21 March 2007; published online 9 May 2007)

\begin{abstract}
We present the design and testing of a rotating device that fits within a commercial helium cryostat and is capable of providing at $4 \mathrm{~K}$ a fresh sample surface for subsequent shots of a $1-10 \mathrm{kHz}$ amplified pulsed laser. We benchmark this rotator in a transient-absorption experiment on molecular switches. After showing that the device introduces only a small amount of additional noise, we demonstrate how the effect of signal degradation due to high fluence is completely resolved. () 2007 American Institute of Physics. [DOI: 10.1063/1.2735557]
\end{abstract}

\section{INTRODUCTION}

When performing transient-spectroscopy experiments on molecular systems with ultrashort, amplified laser pulses, the combination of the fluence (intensity of the light per $\mathrm{cm}^{2}$ ), the absorption, and the dynamics of energy dissipation in the molecules can be such that a fresh sample is required for every incoming laser pulse. Laser pulses may produce undesired long-lived effects such as the formation of triplet states, isomerization of the molecule, or diffusion-limited processes. These slower processes end up drawing more and more molecules from the pool of the accessible ones, with the result of lowering the signal level with each pulse.

At room temperature, with liquids or vapors, there are several techniques that can be used to obtain a fresh sample at every shot, including various types of flow cells, rotating sample cells, and jets. ${ }^{1,2}$ In the low temperature regime, however, where frozen samples form rigid glasses, there seem to be no standard techniques to protect the samples from high fluence and multiple pulses hitting the same area.

Once a sample is frozen in a cryostat, the only two choices to achieve a fresh sample are to either move the laser beams relative to the sample or, conversely, move the sample with respect to the beams. While both solutions are, in principle, feasible, the first option of moving the optical beams presents some obvious technical challenges when two or more beams need to be spatially and temporally overlapped on the sample. Nonetheless, Ionascu et al. recently presented an innovative optical scanning technique for pump-probe spectroscopy, in which an off-axis spinning lens moves the laser beams in a circular trajectory. ${ }^{3}$ Unfortunately when dealing with ultrashort pulses (on the order of $10 \mathrm{fs}$ ) in the visible region, the use of transmissive optics introduces strong chirp. Furthermore, using the same focusing element for both the pump and the probe requires the introduction of separate telescopes for pump and probe to control the relative size of the beams at the sample position. This seems to be an unnecessary complication in the setup and may require more space than available on the optical table.

Moving the sample with respect to the laser beam requires either moving the entire cryostat assembly or, preferably, only the sample. The latter approach was chosen by Larsen who devised a sample holder that could be translated linearly at high speed. ${ }^{4}$ Unfortunately the acceleration/ deceleration at the turning points implies that the motion is not constant.

Here we present the design and testing of a rotating device that fits inside a commercial helium cryostat and is capable of providing a fresh sample surface for subsequent shots of a pulsed laser. In the following of this article we report an example of clear sample degradation due to high fluence. After analyzing the requirements that a sample cell rotator should fulfill, we show how the high-fluence problem is solved by designing a sample cell rotator capable of performing at temperatures as low as $4 \mathrm{~K}$. Finally, after showing

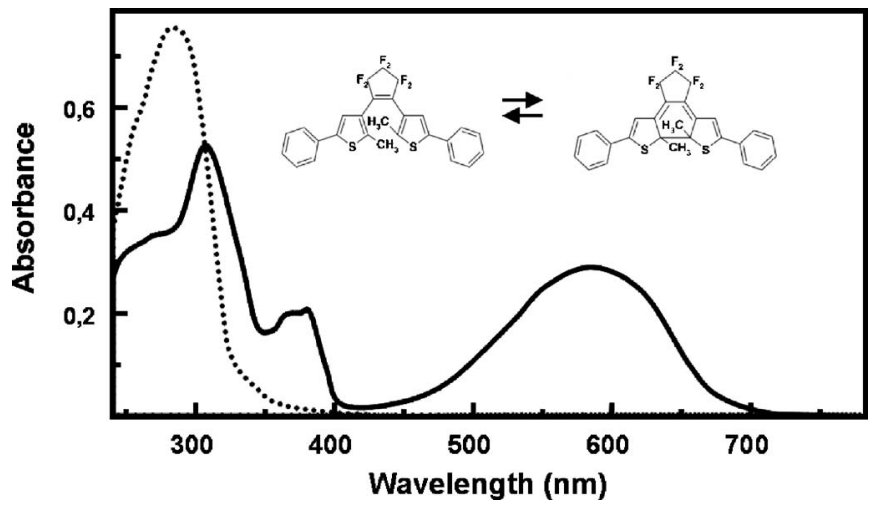

FIG. 1. Absorption spectrum of the molecular-switch B-DTCP used to demonstrate the functioning of our rotating sample holder. Both the absorption of the open form peaking in the UV (dotted line) and the one of the closed form peaking in the visible (solid line) are reported. (Ref. 6). Our laser was tuned to $550 \mathrm{~nm}$. 


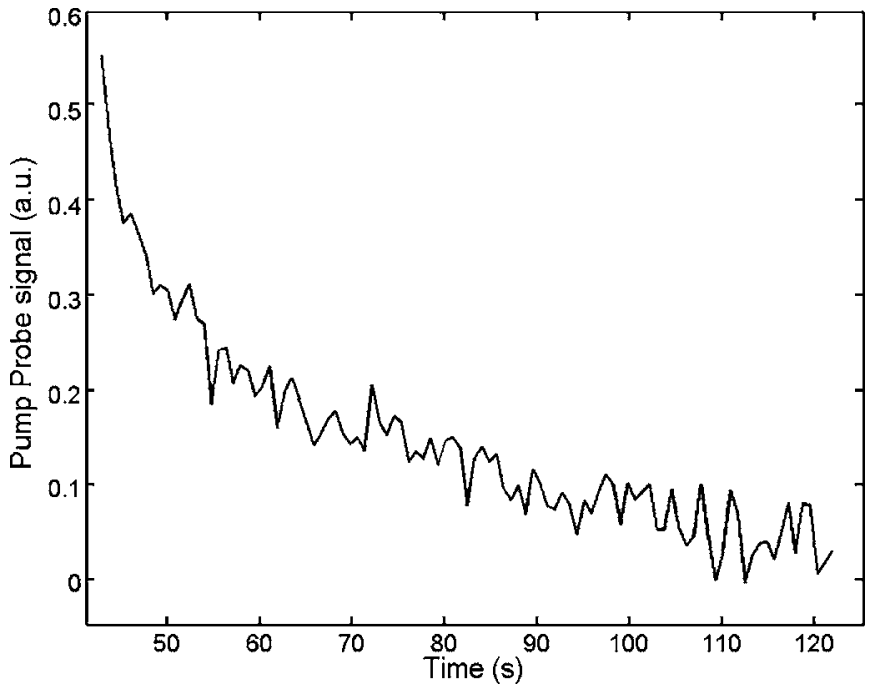

FIG. 2. Pump-probe signal decay due to photoproduct buildup in a stationary sample cell.

that this device introduces only a small amount of additional noise in the measurements, we demonstrate how the effect of signal degradation due to the high fluence (the initial problem that we had set out to solve) is completely resolved.

\section{HIGH FLUENCE AND SAMPLE DEGRADATION: AN EXAMPLE}

When dealing with amplified laser systems producing high energy pulses at high repetition rate $(1-10 \mathrm{kHz})$, in some cases the response of the sample to the absorbed light can last longer than the pulse-to-pulse delay or even be irreversible. This is particularly true, for example, in experiments aimed at coherent control of molecular systems, where high power densities may be needed in order to trigger mul-

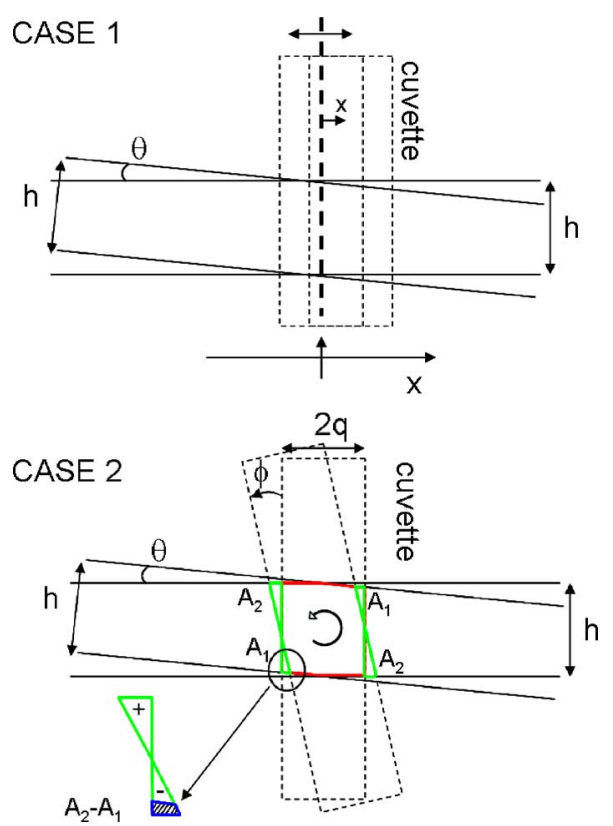

FIG. 3. Representation of the two main cases of instability in the motion of the cuvette leading to an increase in the noise. Case 1: linear vibration perpendicular to the cuvette surfaces. Case 2: rotational motion around the main axis of the cuvette (and of the cryostat rod). tiphoton transitions. ${ }^{5}$ A good example of where this problem seems to arise even at modest fluencies is represented by a class of photoactive molecules commonly referred to as molecular switches. ${ }^{6}$ The optical properties of these molecules change upon absorption of light in the visible region $(500-600 \mathrm{~nm})$. Upon excitation the molecules switch their conformation such that their absorption peak shifts to the UV and the molecules become virtually irresponsive to visible light (see Fig. 1).

When the yield of switched molecules is low enough, this effect can be considered negligible over the duration of the experiment. Upon increasing the laser fluence, however, a significant number of the initial molecules start to disappear from detection, as they have switched to the conformation that absorbs in the UV. As a consequence, if we monitor the absorption over time as the laser beam impinges on the sample, we will find in the transient-absorption signal an induced bleach that will tend to increase over time as the ratio of incident photons over active molecules increases. This effect is clearly displayed in Fig. 2 where the signal drops quickly with a half-life time of a only a few seconds. Note that acquisition times for a pump-probe experiment are typically many minutes.

In order to solve this problem and preserve the active population of our ensemble over the period of a typical pump-probe scan, we needed to move the sample in the cryostat without introducing any significant noise or other signal degradation. To this end, we designed a modified sample rod capable of providing a fresh sample at the repetition rate of the laser system. We are going to take advantage of the properties of such molecules to benchmark the rotator device.

\section{SAMPLE CELL ROTATOR}

\section{A. Design considerations}

Before going in the mechanical details of the cell rotator, the requirements to be fulfilled when designing such a device must be studied.

First of all, one must estimate how fast the sample needs to be moved. With a pump beam of about $150 \mu \mathrm{m}$ in diameter and a laser repetition rate of $1 \mathrm{kHz}$, the sample needs to move away from the laser area with a minimum speed of $15 \mathrm{~cm} / \mathrm{s}$ (to have fresh sample at every shot). Typically sample areas are about $1 \mathrm{~cm}^{2}$ and if a linear motion (easier from a technical perspective) should be used one would need to reverse the direction of motion every 67 laser shots. In a transient-absorption scan the typical averaging is well over this number of shots for each data point. Hence complicated considerations involving the acceleration at the extremes of the linear motion would have to be made. ${ }^{4}$ Alternatively a circular motion, with constant speed, will take care of this problem.

Given a circular geometry of the sample path, we then assess the stability requirements of the motion for it to be considered a low-noise device ( $\leqslant 1 \%$ of the signal). We will need to maintain constant both the fluence and the spatial overlap of the beams in the sample volume. How critical this aspect of the motion is depends on the relative angle of the incoming beams as well as on their depth of focus. Within a 


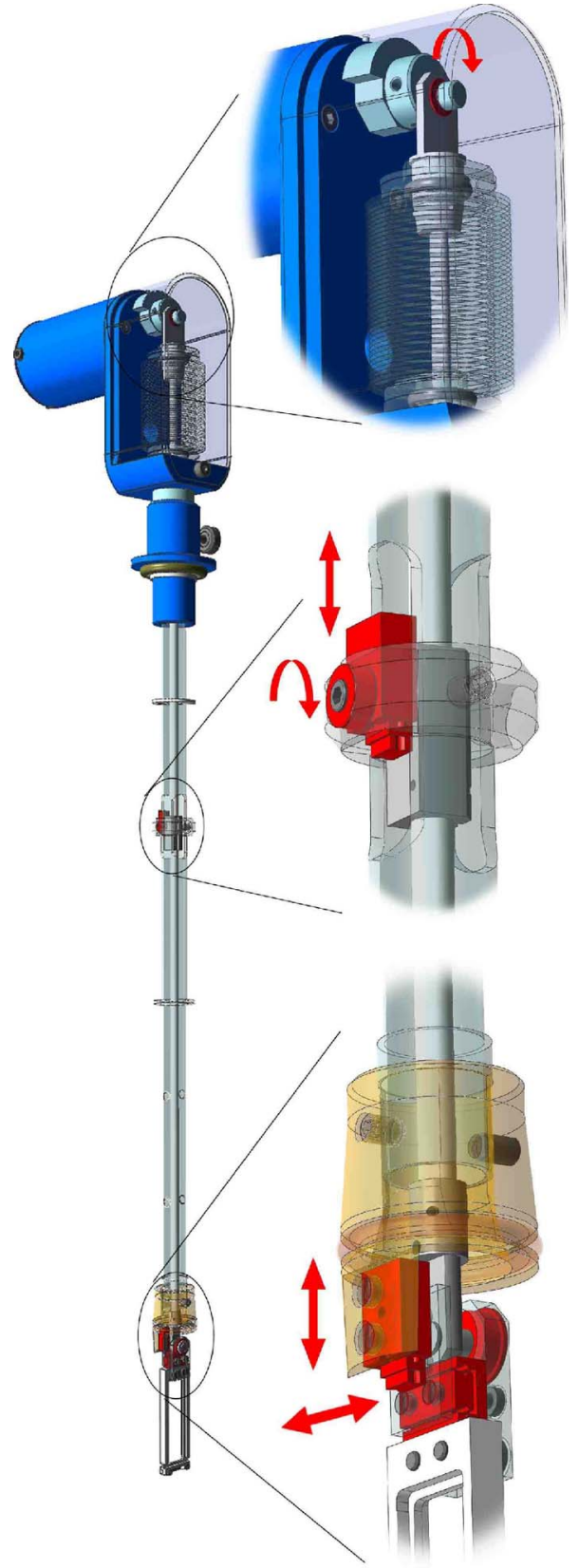

FIG. 4. Schematic representation of the sample rod rotator. Highlighted regions show details of the design. Top: the eccentric positioning of the crank to the motor provides variable diameters in sample motion. The gastight metal bellow provides flexible motion and thermal insulation. Middle: ball bearing. Bottom: The x-y linear bearings ensure strict in plane motion.

cylindrical beam approximation we estimated the precision required for the motion of the cuvette. Two off-plane motions of the cuvette could introduce a change in the active volume (volume where sample, pump beam, and probe beam overlap) that could lead to noise. One is a vibrational motion of the cuvette in the direction perpendicular to its surface (Fig. 3, case 1) and the other is a rotation of the cuvette around the axis of the cryostat sample rod (Fig. 3, case 2). Approaching the linear motion first, one can calculate first the change in active volume for a given shift $x$ of the center of the cuvette with respect to the plane of intersection of the beams (Fig. 3, case 1). If we define $2 q$ to be the cuvette thickness, $h$ to be the diameter of (both) beams, and $\theta$ to be

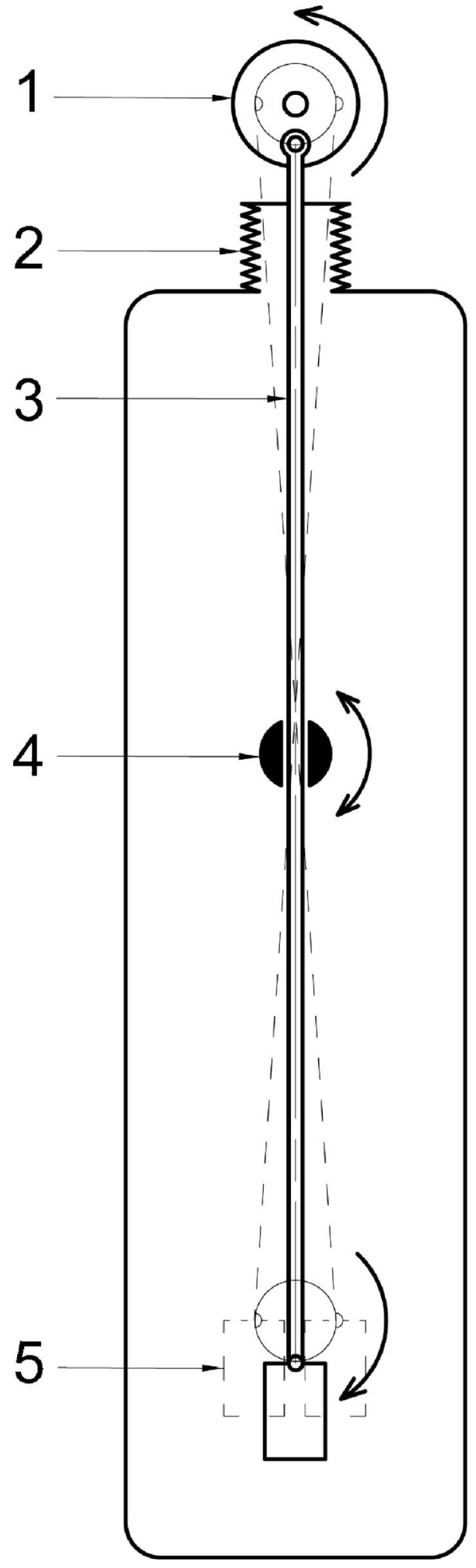

FIG. 5. Schematic of the rotating sample holder. 1: electric motor, 2: gastight bellow, 3: ceramic rod, 4: ball bearing, 5: x-y linear bearings.

the angle between them, then the active volume will vary with $x$ according to

$$
V(x)=2 q h^{2}-\frac{\sin (\theta)}{2} h q^{2}-h x^{2} .
$$

Using our numbers for $2 q(500 \mu \mathrm{m}), h(150 \mu \mathrm{m})$, and $\theta$ $\left(5^{\circ}\right)$, one finds that a $1 \%$ change in active volume corre- 
sponds to an off-plane cuvette shift of about $160 \mu \mathrm{m}$. The effect of the cuvette rotation around its longer axis is explored in Fig. 3, case 2. The analytical form of the change in the active volume is too cumbersome to report, hence, for simplicity we show in Fig. 3, case 2 (inset) where the change in the active volume arises by showing, after a rotation by an arbitrary angle $\phi$, the difference (shaded area) between the gained active volume (marked as "+") and the lost active volume (marked as "-"). After going through some simple geometrical analysis, one finds that using the same numbers reported above, an angle $\phi$ equal to about $15^{\circ}$ is required to introduce a change of $1 \%$ in the active volume. Summarizing, we have found that one needs a device to move the sample in a circular motion, with a speed of at least $15 \mathrm{~cm} / \mathrm{s}$ and a necessary in-plane and torsional stability at least of the order of $160 \mu \mathrm{m} 15^{\circ}$, respectively.

\section{B. Rotator description}

Having seen the type of problems (Fig. 2) that a fixed sample cell can produce and the requirements that a sample translator should fulfill in order to solve those problems, we now describe in detail our new design.

Starting from a commercially available cryostat (Oxford OptistatCF), we designed a new sample rod (Fig. 4) capable of transferring the motion from an electric motor placed at the top end of the rod (at room temperature) to the sample holder down in the sample chamber of the cryostat at temperatures as low as $4 \mathrm{~K}$. A linkage mechanism is used, rather than a linear actuator. The advantages of this design over a more conventional one with an actuator are numerous: it enables continuous motion, higher and constant speed, minimal vibrations, and minimal instrument size and weight. Schematic drawings of the sample rod rotator are shown in Figs. 4 and 5 . The oscillator is powered by a geared electric motor $(400 \mathrm{rpm})$ that is located at the top of the rod outside the cryostat (point 1 in Fig. 5). The output shaft of the motor is fitted with a crankshaft shown in detail in Fig. 4 (top). The eccentric position of the crank with respect to the motor has four settings that yield four different diameters in the sample motion $(2,3,4$, and $5 \mathrm{~mm})$. To minimize vibrations the crankshaft is balanced by a counterweight.

The crank drives a ceramic rod to ensure rigidity and thermal insulation. This rod runs first through a gas-tight bellow (point 2 in Fig. 5) that can be filled with $\mathrm{He}$ gas to avoid the forming of frost. The rod runs then through a cylinder (point 3 in Fig. 5), reaching all the way down to the sample holder. The rod is held in the middle by a miniature linear bearing rotating in a ball bearing to increase stability (point 4 in Fig. 5). Both ends of the rod rotate in ball bearings [see Fig. 4 (middle)]. Finally, at the bottom end of the rod, the sample holder is guided in its in-plane motion by two miniature linear bearings mounted perpendicularly to each other (point 5 in Fig. 5). This final linear guiding, shown in detail in Fig. 4 (bottom), ensures a stable, two dimensional, movement at the sample position. It also prevents the sample windows from rotating around an axis perpendicular to the windows of the cuvette. The sample can be placed in a normal static cuvette or between windows of

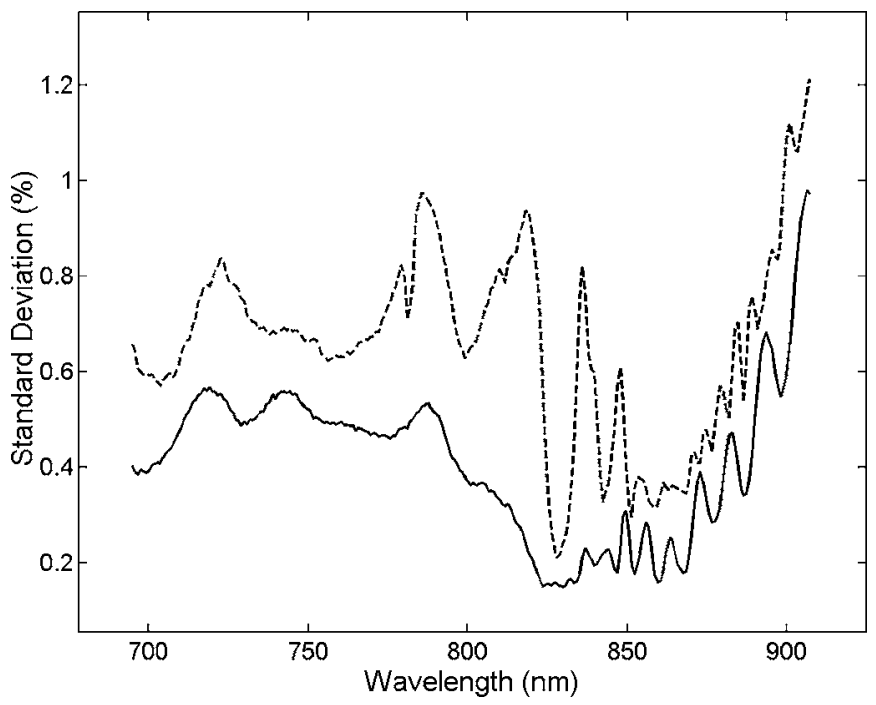

FIG. 6. Standard deviation (as percent of the pump-probe signal) of the spectrally dispersed pump-probe signal. The solid curve corresponds to the rotator-off case and the dashed line corresponds to the rotator-on case.

different materials (glass, quartz, or plastic) that are fitted into a Teflon frame [Fig. 4 (bottom)].

\section{TESTING OF THE ROTATOR AND FINAL COMMENTS}

First, we checked that all the moving parts in our device were functioning at low temperature $(4 \mathrm{~K})$ without affecting the performance of the cryostat. No noticeable increase in liquid $\mathrm{He}$ consumption was observed due to friction from the moving parts.

Next, the stability of the sample cell motion and the effect of the rotation on the noise level of our pump-probe signal were checked. The first test consisted in shining a $\mathrm{He}-\mathrm{Ne}$ laser beam (1 $\mathrm{mm}$ diameter) on the sample cell and observing the position of the reflected beam on a screen far from the cryostat as we powered the rotator. The input angle of the beam (with respect to the normal to the surface of the sample cell) was $14^{\circ}$ and the screen, where the reflected

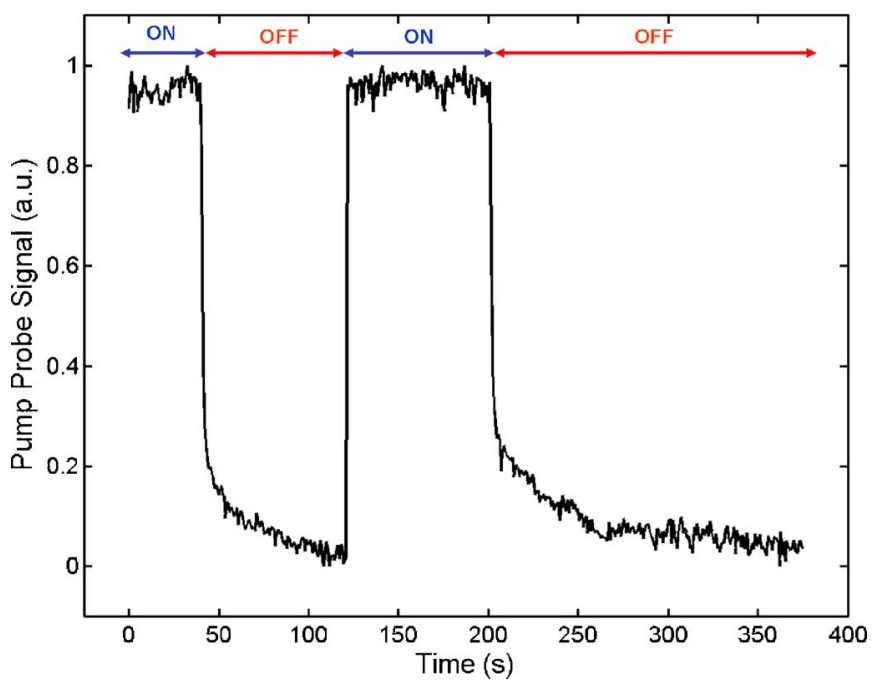

FIG. 7. Decay of the signal vs time (rotator off) and immediate recovery of the signal when the rotator is powered on. 
beam was monitored, was placed $1 \mathrm{~m}$ away from the sample cell. When rotating the cell, we observed a shifting of the beam position within a radius of about $450 \mu \mathrm{m}$, independent of the speed of the rotator. This displacement seems to point to a range of off-plane oscillation of the sample cell of about the same order. Notice that if we refer to our previous geometrical estimate, we should expect a negligible increase in the noise.

In order to check our estimate, we then proceeded to measuring a pump-probe signal and calculate its standard deviation over a fixed number of shots (1000 shots or $1 \mathrm{~s}$ integration time) from our laser. This method gives us a direct measure of the signal noise over an integration time typical in pump-probe experiments. As shown in Fig. 6, the difference in the noise level is about $0.2 \%$ over the whole spectrum (measured from 680 to $920 \mathrm{~nm}$ ). Variations of the noise across the spectrum are due to spectral instabilities in the white light used as the probe pulse.

Finally, we return to the molecular-switch sample whose rapid signal decay is used to benchmark the usefulness of the rotator (Fig. 7). This represents the final and most important test, where we actually verify directly the effectiveness of our device in providing a fresh sample spot at every laser shot (corresponding to a speed in excess of $15 \mathrm{~cm} / \mathrm{s}$ ) without affecting noticeably the signal quality. In Fig. 7 we show the effect of powering on and off the rotator on the signal. As it can be clearly observed, the signal degradation is now completely under control by applying the rotator. We conclude by remarking that this approach is applicable to any cryostat where the sample sits in exchange gas and is positioned by an externally adjustable rod.

\section{ACKNOWLEDGMENTS}

The authors would like to acknowledge the technical staff of AMOLF for their invaluable help in making a prototype of the rotator, software engineer Niels Dijkhuizen for assistance with the LABVIEW code used to run the tests, $\mathrm{Ad}$ de Snaijer for the cryotesting of some parts, and Maaike Milder for general assistance with the samples. This work is part of the research program of the "Stichting voor Fundamenteel Onderzoek der Materie (FOM)," which is financially supported by the "Nederlandse organisatie voor Wetenschappelijk Onderzoek (NWO)."

\footnotetext{
${ }^{1}$ J. Bredenbeck and P. Hamm, Rev. Sci. Instrum. 74, 3188 (2003).

${ }^{2}$ M. M. Hoffmann, R. S. Addleman, and J. L. Fulton, Rev. Sci. Instrum. 71, $1552(2000)$

${ }^{3}$ D. Ionascu, F. Rosca, F. Gruia, A. Yu, and P. M. Champion, Rev. Sci. Instrum. 77, 64303 (2006).

${ }^{4}$ D. S. Larsen, Rev. Sci. Instrum. 73, 1325 (2002).

${ }^{5}$ M. Shapiro and P. Brumer, Adv. At., Mol., Opt. Phys. 42, 287 (2000).

${ }^{6}$ B. Feringa, Molecular Switches (Wiley-VCH, Weinheim, 2001).
} 